\title{
Cribado: para qué y cómo
}

\section{Screening: why and how}

Nieves Ascunce Elizaga

El cribado, en el marco de los sistemas sanitarios, se refiere a la realización de pruebas diagnósticas a personas, en principio sanas, para distinguir aquellas que probablemente estén enfermas de las que probablemente no lo están. Se trata de una actividad de prevención secundaria, cuyo objetivo es la detección precoz de una determinada enfermedad a fin de mejorar su pronóstico y evitar la mortalidad prematura y/o la discapacidad asociada a la misma. Pero si también es posible la detección de lesiones o situaciones previas a la aparición de la enfermedad en cuestión, su tratamiento permitirá además reducir su incidencia.

Cribado, detección precoz, diagnóstico precoz, son actualmente términos muy populares. La población reclama pruebas de cribado y los sanitarios las ofertan. Parece que detectar pronto una enfermedad es garantía de curación, éxito seguro. Pero el cribado, como toda actividad sanitaria tiene beneficios, pero también efectos adversos. Además de los potenciales efectos secundarios asociados a las pruebas y al tratamiento, los pacientes en los que la detección precoz no suponga una mejora en su pronóstico sufrirán un periodo de morbilidad mayor por el adelanto diagnóstico que el propio proceso implica. La detección de anomalías de pronóstico incierto o lesiones precursoras puede derivar en sobre-diagnósticos y sobre-tratamientos.

Por estos y otros muchos motivos que se podrían considerar, evidentemente el cribado no es una panacea y ni mucho menos está justificado en sí mismo.

Para empezar, no todas las enfermedades son susceptibles de control mediante esta práctica. Los criterios a este respecto son claros: la enfermedad debe ser un importante problema de salud, con una historia natural bien definida y conocida etc. Tampoco se puede ni se debe considerar cualquier tipo de prueba; un test de cribado debe ser válido, fiable, simple, seguro y aceptable por la población a la que va dirigi-

Médico Especialista en Medicina Preventiva y

Salud Pública

Instituto de Salud Pública y Laboral de Navarra.

Pamplona

CIBER de Epidemiología y Salud Pública

\section{Correspondencia:}

Nieves Ascunce

Instituto de Salud Pública y Laboral de Navarra.

C/ Leyre, 15

31003 Pamplona. Navarra

E-mail: nascunce@navarra.es 
do. Debe haber un claro consenso respecto al proceso de confirmación diagnóstica y evidencia de que el tratamiento en fase presintomática es más efectivo ${ }^{1}$.

Pero además de todo esto, antes de recomendar un cribado, la eficacia en la reducción del riesgo de mortalidad o morbilidad debe estar claramente demostrada en estudios científicos de calidad y no solo eso, sino que los resultados de esos estudios deben poderse extrapolar al contexto real en el que se va a implantar y desarrollar el programa de cribado que se está considerando. Pero, como ya se ha comentado, también se deberán valorar sus potenciales efectos adversos y demostrar que en el balance riesgo/beneficio, el resultado es positivo.

En base a esos criterios y a los resultados de impacto de los numerosos programas en desarrollo no solo en España sino en Europa, diversos organismos e instituciones han elaborado recomendaciones sobre las enfermedades que pueden abordarse a través de programas de cribado. A modo de ejemplo, y en relación con el cribado de cáncer, el Consejo de la Unión Europea y la estrategia del cáncer del SNS recomienda que se desarrollen programas de cribado de cáncer de mama, de cuello de útero y de colon y recto especificando la población objetivo y test de cribado que debe utilizarse ${ }^{2,3}$. Para el resto de población y para el resto de tumores malignos, no hay suficiente evidencia de que el cribado produzca un beneficio que compense los efectos adversos que podría suponer, por lo que no se justifica su realización, ni desde el punto de vista individual ni poblacional.

Recientemente se ha publicado una actualización de la cartera de servicios comunes del Sistema Nacional de Salud en la que recoge como prestación básica los cribados para cáncer de mama, cérvix y colon y recto ${ }^{4}$.

Pero tanto en esta actualización como en todas las mencionadas anteriormente se establece la necesidad de que los cribados se realicen en el marco de programas organizados de carácter poblacional. El cribado oportunista nunca se debe considerar como una alternativa al poblacional cuando este no ha demostrado ser eficaz, y esto es algo que se debe tener en cuenta a la hora de ofertar en la práctica clínica habitual pruebas de cribado

Un cribado se considera cribado poblacional cuando esta actividad preventiva se aplica a todas las personas residentes en la comunidad, independientemente del sistema de aseguramiento que cada persona tenga o de cualquier otra circunstancia individual o social, de manera sistemática, con invitación individual de cada persona de la población que se haya definido como objetivo. Es un proceso organizado e integrado en el sistema de salud, en el que todas las actividades están planificadas, coordinadas, monitorizadas y evaluadas dentro de un marco de mejora continua de la calidad, garantizando los principios de eficiencia y equidad. Solo de esta manera se conseguirá obtener el beneficio que el proceso conlleva minimizando los efectos adversos inherentes al propio proceso. La realización del cribado en este marco es la forma de garantizar que los efectos beneficiosos superan significativamente a los adversos que también se derivarán del mismo. En España, siguiendo esta recomendación, se han puesto en marcha programas de cribado de cáncer de mama en todas las CCAA y se están implantando 
de colon y recto progresivamente ${ }^{5,6}$. Pero el cribado organizado y de carácter poblacional además también tiene otras ventajas.

El trabajo que se publica en este mismo número sobre desigualdades sociales en el cribado de cáncer de cérvix y mama en la comunidad de Madrid ${ }^{7}$ analiza la práctica de citología y mamografía en dicha comunidad según nivel educativo y clase social. Aunque no se analiza dónde se han realizado estas pruebas, hay una clara diferencia entre ellas; la citología siempre se habrá realizado con carácter individual y de forma oportunista y la mamografía al menos cabe la posibilidad de que se haya realizado en el marco del programa poblacional que la comunidad oferta. Es significativa su conclusión sobre el hallazgo de un gradiente social para las prácticas preventivas, menor en la práctica de la mamografía (programa poblacional) que en la citología (actividad de carácter oportunista)

Los programas organizados de carácter poblacional, con la oferta de realización de la exploración a toda la población garantizan el principio de equidad, pero además suelen integrar actividades de sensibilización especial y formas de acceso también especiales para grupos especialmente desfavorecidos, grupos que de entrada suelen tener una participación menor. De esta forma se consigue disminuir ese gradiente social que de por si siempre se produce en la práctica de actividades preventivas.

\section{BIBLIOGRAFÍA}

1. Viñes Rueda J.J. La efectividad de la detección precoz de enfermedades An Sist Sanit Navar 2007; 30: 11-27.

2. Comisión de la Comunidad Europea. Propuesta de Recomendación del Consejo sobre cribado de cáncer. Bruselas, 5.5. 2003. Volumen 2003/878/CE, 2003:L327/34-L327/37. Disponible en http://ec.europa.eu/health

3. Estrategia en Cáncer en el Sistema Nacional de Salud. Actualización 2009. Madrid: Ministerio de Sanidad y Política Social. Disponible en http://www. msssi.gob.es/organizacion/sns/planCalidadSNS/pdf/ActualizacionEstrategiaCancer.pdf

4. Orden SSI/2065/2014, de 31 de octubre, por la que se modifican los anexos I, II y III del Real Decreto 1030/2006, de 15 de septiembre, por el que se establece la cartera de servicios comunes del Sistema Nacional de Salud y el procedimiento para su actualización. BOE Núm. 269, 6 de noviembre de 2014. http://www.boe.es

5. Red de Programas de Cribado de Cáncer. http://www.cribadocancer.com/

6. Ascunce N, Barcos A, Ederra M, Erdozain N, Murillo A, Osa A et al. Programa de detección precoz de cáncer de mama. Resultados de proceso e indicadores de impacto (1990-2002). An Sist Sanit Navar 2004; 27: 319-333.

7. Latasa P, Gandarillas AM, Ordobás M. Tendencias y desigualdades sociales en el cribado de cáncer de cérvix y cáncer de mama en la Comunidad de Madrid durante el periodo 1995-2010 a partir del Sistema de Vigilancia de Factores de Riesgo (Sivfrent-A). An Sist Sanit Navar 2015; 38: 21-30. 
